\title{
ON THE ROLE OF L-BAIRE FUNCTIONS IN ABSTRACT MEASURE AND INTEGRATION
}

\author{
by MELVIN J. MARON
}

(Received 2 September, 1974)

1. Introduction. If $X$ is any set and $L \subset[-\infty, \infty]^{X}$, the class $\mathscr{B}_{L}$ of $L$-Baire functions is defined to be the smallest subclass of $[-\infty, \infty]^{X}$ which contains $L$ and is closed under the formation of monotone, pointwise, sequential limits, so that $\mathscr{B}_{L} \ni f_{n} \nearrow f$ or $\mathscr{B}_{L} \ni f_{n} \searrow f \Rightarrow$ $f \in \mathscr{B}_{L}$.

Segal and Kunze [7], following Loomis [4], used this idea as the basis for a very efficient, elementary presentation of the theory of measure spaces $(X, \mathbf{S}, \mu)$ and their associated integral spaces $\left(X, \mathscr{L}(\mathbf{S}, \mu), \int \cdot d \mu\right)$. Maron [5] then used it to study (not necessarily constructed) abstract integral spaces $(X, \mathscr{L}, I)$ in the absence of any structure on $X$.

In [7], measurable functions and constructed integrals are presented in an "integral oriented" way to illuminate the role of $L$-Baire functions. In this article we show how to use $L$-Baire functions to give quick, informative proofs of the basic properties of measures and their associated integrals (on the class of summable, measurable functions) using the "measure oriented" definitions of Halmos [2]. The idea is to show that the objects defined in the "measure oriented" way coincide with an "integral oriented" counterpart, hence a fortiori have the desired properties (see (2.5), (2.6) and (5.5)).

Having done this, it is easy to obtain a very sharp and general Riesz-Markov type theorem (6.3) which describes the 1-1 correspondence between the collection of all (not necessarily complete) $\sigma$-finite measure spaces $(X, \mathrm{~S}, \mu)$ and the collection of all integral spaces $(X, \mathscr{L}, I)$

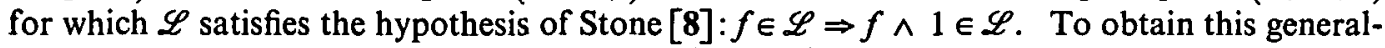
ity, we use the definition of an integral space $(X, \mathscr{L}, I)$ given in [5] which avoids null sets by allowing $\mathscr{L} \subset[-\infty, \infty]^{x}$.

A discussion of the results obtained and their proofs is given in $\S 7$.

2. Preliminaries on collections of sets and functions. The symbols $R$ and $[-\infty, \infty]$ denote respectively the set of real and extended real numbers. We take $0 \cdot( \pm \infty)=0$ and leave $\pm(\infty-\infty)$ undefined. The order and algebraic operations, and limits of sequences in $[-\infty$, $\infty]^{X}$ are defined in the natural (pointwise) way. A function lattice (on $X$ ) is a nonempty class $\mathscr{L} \subset[-\infty, \infty]^{X}$ which is closed under (real) scalar multiplication and under the lattice operations (i.e. $f \vee g=\max \{f, g\}$ and $f \wedge g=\min \{f, g\}$ are in $\mathscr{L}$ whenever $f, g \in \mathscr{L}$ ), and which is conditionally closed under addition in the sense that $f+g$ is in $\mathscr{L}$ whenever $f, g \in \mathscr{L}$ and $f+g$ is defined (at each point of $X$ ); if it happens that $\mathscr{L} \subset R^{X}$ then $\mathscr{L}$ is called a Riesz space or real function lattice. Since $f+g$ is defined for any $f, g \in R^{X}$, a Riesz space is simply a vector space of functions in $R^{X}$ which is closed under the lattice operations.

The most important result about $L$-Baire functions for our purposes is Theorem 9 of [5] which we now restate for reference. 
(2.1) Monotone Class Lemma for Functions. If $L$ is a Riesz space then $\mathscr{B}_{L}$ is a function lattice.

Throughout, $\mathbf{R}$ will denote a ring and $\mathbf{S}$ a $\sigma$-ring of subsets of $X, \mathbf{S}(\mathbf{R})$ will denote the $\sigma$-ring generated by $\mathbf{R}$, and $\chi_{A}$ will denote the characteristic function of $A \subset X$. Then the following result is immediate from (2.1).

(2.2) Lemma. IfL is a Riesz space then the class $\left\{A \subset X: \chi_{A} \in \mathscr{B}_{L}\right\}$ is a $\sigma-r i n g$.

Recall that $f \in[-\infty, \infty]^{X}$ is called S-measurable if

$$
f^{-1}(c, \infty] \in \mathbf{S} \text { and } f^{-1}[-\infty,-c) \in \mathbf{S} \text { for all } c \in(0, \infty) \text {. }
$$

We denote by $\mathscr{M}_{\mathrm{S}}$ the class of S-measurable functions. Another important class of functions is the Riesz space $L[\mathbf{R}]$, which consists of the R-simple functions, i.e. those $s \in R^{X}$ which can be represented (not uniquely) as a finite sum

$$
s=\sum_{i} a_{i} \chi_{A_{i}}, \quad \text { where } \quad a_{i} \in R \text { and } A_{i} \in \mathbf{R} .
$$

(2.5) Proposition. Iff $\in[0, \infty]^{X}$, the following are equivalent:

(i) $f \in \mathscr{M}_{\mathbf{S}(\mathbf{R})}$, i.e.fis $\mathbf{S}(\mathbf{R})$-measurable.

(ii) There exist $s_{n} \in L[\mathbf{S}(\mathbf{R})]$ such that $0 \leqq s_{n} / f$, i.e. $0 \leqq s_{1} \leqq s_{2} \leqq \ldots$ and $f=\sup _{n} s_{n}=$ $\lim _{n} s_{n}$.

(iii) $f \in \mathscr{B}_{L[\mathbf{S}(\mathbf{R})]}$, i.e. fis an $L[\mathbf{S}(\mathbf{R})]$-Baire function.

(iv) $f \in \mathscr{B}_{L[\mathbf{R}]}$, i.e. fis an $L[\mathbf{R}]$-Baire function.

Proof. (i) $\Rightarrow$ (ii) is standard (see [2] Theorem 29B p. 85), and (ii) $\Rightarrow$ (iii) is true by the definition of $\mathscr{B}_{L[\mathbf{S}(\mathbf{R})]}$.

(iii) $\Rightarrow$ (iv). Since $\left\{A \subset X: \chi_{A} \in \mathscr{B}_{L[\mathrm{R}]}\right\}$ is a $\sigma$-ring (Lemma (2.2)) which contains $\mathbf{R}$, it contains $\mathbf{S}(\mathbf{R})$. But $\mathscr{B}_{L[R]}$ is a function lattice, by the Monotone Class Lemma (2.1); so it follows from (2.4) that every $s \in L[\mathbf{S}(\mathbf{R})]$ is an $L[\mathbf{R}]$-Baire function. But $\mathscr{B}_{L[R]}$ is, by definition, closed under the formation of monotone sequential limits; so it must contain every $L[S(R)]-$ Baire function.

(iv) $\Rightarrow$ (i). Observe that for any $c \in(0, \infty),\left\{f \in[-\infty, \infty]^{X}: f^{-1}(c, \infty] \in \mathbf{S}(\mathbf{R})\right\}$ is a monotone class that contains $L[\mathbf{R}]$ (see [7], Theorem 3.1 p. 52).

For any class $\mathscr{F} \subset[-\infty, \infty]^{x}$, define $\mathscr{F}^{+}=\{f \in \mathscr{F}: f \geqq 0\}$.

If $f \in[-\infty, \infty]^{X}$ then by $(2.3), f \in \mathscr{M}_{\mathrm{s}}$ if and only if both $f^{+}=f \vee 0$ and $f^{-}=(-f) \vee 0$ are in $\mathscr{M}_{\mathbf{S}}^{+}$. The following elementary properties of the class $\mathscr{H}_{\mathbf{S}}$ are therefore immediate from (2.5) and (2.1):

(2.6) CoRollary. $\mathscr{M}_{\mathrm{S}}=\mathscr{B}_{L[\mathrm{~S}]}$, hence $\mathscr{M}_{\mathrm{S}}$ is a function lattice. Moreover, $\mathscr{H}_{\mathrm{S}}$ is the smallest class in $[-\infty, \infty]^{X}$ which contains $\chi_{A}$ for each $A \in \mathbf{S}$ and which is closed under the formation of monotone sequential limits. 
3. Relevant facts about integrals. An elementary integral space is a triple $(X, L, I)$, where $L$ is a Riesz space of functions on $X$ and $I$ is an elementary integral on $L$, i.e. a positive linear functional on $L$ such that $L \ni f_{n} \searrow 0 \Rightarrow I f_{n} \searrow 0$. An integral is a functional $I: \mathscr{L} \rightarrow R$, (where $\mathscr{L}$ is a function lattice on $X$ ), which is monotone and conditionally linear in the sense that if $f, g \in \mathscr{L}$ and $a, b \in R$ and $a f+b g$ is defined (on $X)$, then $I(a f+b g)=a I f+b I g$; and which satisfies the Monotone Convergence Theorem, namely

$$
\mathscr{L} \ni f_{n} \nearrow f \text { and } \lim _{n} I f_{n}<\infty \Rightarrow f \in \mathscr{L} \text { and If }=\lim _{n} I f_{n} .
$$

In this case the triple $(X, \mathscr{L}, I)$ is called an integral space.

The notation $(X, \mathscr{L}, I) \leqq(X, \hat{\mathscr{L}}, \mathcal{I}),(\operatorname{read}(X, \mathscr{L}, \mathcal{I})$ extends $(X, \mathscr{L}, I))$, means $\mathscr{L} \subset \hat{\mathscr{L}}$ and $I$ extends $I$. Uniqueness of integral extensions is assured by the following important result (Theorem 8 of [5]):

(3.2) UNIQUeNess Theorem. Suppose that $(X, \mathscr{L}, I)$ and $(X, \hat{\mathscr{L}}, I)$ are integral spaces and that $L$ is a function lattice contained in $\mathscr{L} \cap \hat{\mathscr{L}}$ such that the restrictions of $I$ and $\mathcal{I}$ to $L$ coincide. Then for any $f \in \mathscr{B}_{L}^{+}$, either $f \in \mathscr{L} \cap \hat{\mathscr{L}}$ and If $=$ If or there exist $f_{n} \in \mathscr{L} \cap \hat{\mathscr{L}}$ such that $0 \leqq f_{n} \nearrow$ fand $\lim _{n} I f_{n}=\lim _{n}\left\{f_{n}=\infty\right.$.

If the integral $I$ is defined on the function lattice $\mathscr{L}$, it will be convenient to extend $I$ to all of $\mathscr{B}_{\mathscr{L}}^{+}$by taking If $=\infty$ whenever $f \in \mathscr{B}_{\mathscr{L}}^{+} \mid \mathscr{L}$; we are then assured by (3.1) and (3.2) that for any $f, g, f_{n} \in \mathscr{B}_{\mathscr{L}}^{+}$,

$$
f \leqq g \Rightarrow I f \leqq I g, \text { and } I\left(\sum_{n} f_{n}\right)=\sum_{n} I f_{n} .
$$

Integral extensions are assured by the following result:

(3.4) ExistenCe THEOREM. Let $(X, L, I)$ be an elementary integral space. (a) Using the Daniell extension procedure (see $\S 3.3$ of [7] for details) one can construct an integral space $\left(X, \mathscr{L}_{I}, I\right)$ which extends $(X, L, I)$. (b) The restriction of the integral I of (a) to the function lattice $\mathscr{B}_{L} \cap \mathscr{L}_{1}$ is clearly an integral. The resulting integral space $\left(X, \mathscr{D}_{L} \cap \mathscr{L}_{I}, I\right)$, called the Baire extension of $(X, L, I)$, is minimal in the sense that for any integral space $(X, \hat{\mathscr{L}}, \hat{I})$ we may take $\mathscr{L}=\mathscr{L}_{I}$ in (3.2) to conclude that

$$
(X, L, I) \leqq(X, \hat{\mathscr{L}}, \hat{I}) \Rightarrow\left(X, \mathscr{B}_{L} \cap \mathscr{L}_{I}, \hat{I}\right) \leqq(X, \hat{\mathscr{L}}, \hat{I}) .
$$

4. The integral space associated with an elementary measure. The map $\mu: \mathbf{R} \rightarrow[0, \infty]$ is called an elementary measure on $\mathbf{R}$, and the triple $(X, \mathbf{R}, \mu)$ is called an elementary measure space if $\mu(\emptyset)=0$ and $\mu$ is countably additive, i.e. if $A_{n} \in \mathbf{R}(n=1,2, \ldots)$ are pairwise disjoint and if $\bigcup_{n} A_{n} \in \mathbf{R}$, then $\mu\left(\bigcup_{n} A_{n}\right)=\sum_{n} \mu\left(A_{n}\right)$. Both $\mu$ and $(X, \mathbf{R}, \mu)$ are called $\sigma$-finite if every $A \in \mathbf{R}$ is of $\sigma$-finite $\mu$-measure. Evidently, an elementary measure is a measure if and only if it is defined on a $\sigma$-ring.

Let $(X, \mathbf{R}, \mu)$ be an elementary measure space. Then the class

$$
\mathbf{R}_{\mu}=\{A \in \mathbf{R}: \mu(A)<\infty\}
$$


is also a ring, and the real number $I_{\mu} s$, given by

$$
I_{\mu} s=\sum a_{i} \mu\left(A_{i}\right) \text { for } s \in L\left[\mathbf{R}_{\mu}\right]
$$

as in (2.4), unambiguously defines an elementary integral $I_{\mu}$ on the Riesz space $L\left[\mathbf{R}_{\mu}\right]$ ( $[9]$ Theorem 1, p. 75), so $\left(X, L\left[\mathbf{R}_{\mu}\right], I_{\mu}\right)$ is an elementary integral space. Its Baire extension (3.4(b)) is denoted by $\left(X, \mathscr{L}_{\mu}[\mathbf{R}], I_{\mu}\right)$ and will be called the minimal integral space associated with $(X, \mathbf{R}, \mu)$. Note that by $(3.4(\mathrm{~b})$ and $(2.5)$

$$
\mathscr{L}_{\mu}[\mathbf{R}] \subset \mathscr{B}_{L\left[\mathbf{R}_{\mu}\right]}=\mathscr{M}_{S\left(\mathbf{R}_{\mu}\right)} .
$$

The familiar extension theorem for measures ([2] Theorem 13A, p. 54) can now be deduced from the existence of $\left(X, \mathscr{L}_{\mu}[\mathrm{R}], I_{\mu}\right)$.

(4.4) THEOREM. Let $\mu$ be a $\sigma$-finite elementary measure on the ring $\mathbf{R}$. Then by taking $\hat{\mu}(A)=I_{\mu}\left(\chi_{A}\right)$ for $A \in \mathbf{S}(\mathbf{R})$ we get the unique measure $\hat{\mu}$ on $\mathbf{S}(\mathbf{R})$ extending $\mu ; \hat{\mu}$ is $\sigma$-finite.

Proof. See Corollary 3.5.1 of [7].

5. The minimal integral space as a constructed integral. The integral space $\left(X, \mathscr{L}_{\mu}[\mathbf{R}]\right.$, $\left.I_{\mu}\right)$ associated with the elementary measure space $(X, \mathbf{R}, \mu)$ in $\S 4$ was obtained quickly because we used the rather elaborate, general Baire extension. If we are given a (not necessarily $\sigma$-finite) measure space $(X, \mathbf{S}, \mu)$, a more direct construction of an integral proceeds as follows:

DEFINITION. For any $s \in L[\mathrm{~S}]^{+}$represented as in (2.4), take (5.1)

$$
\int s d \mu=\sum_{i} a_{i} \mu\left(A_{i}\right) \text {. }
$$

(Bear in mind our convention that $0 \infty=0$.) Then $\int s d \mu \in[0, \infty]$ and, as in $\S 4$, is independent of the representation (2.4) for $s \in L[\mathrm{~S}]^{+}$. For any $f \in \mathscr{M}_{\mathrm{S}}^{+}$, define $\int f d \mu(\in[0, \infty])$ by

$$
\int f d \mu=\sup \left\{\int s d \mu: f \geqq s \in L[\mathbf{S}]^{+}\right\} .
$$

Finally, define the family $\mathscr{L}(\mathbf{S}, \mu)$ of $\mu$-summable functions to consist of those $f \in \mathscr{M}_{\mathbf{S}}$ such that $\int f^{+} d \mu<\infty$ and $\int f^{-} d \mu<\infty$, and define the functional $\int \cdot d \mu$ on $\mathscr{L}(\mathbf{S}, \mu)$ by

$$
\int f d \mu=\int f^{+} d \mu-\int f^{-} d \mu, \text { for } f \in \mathscr{L}(\mathbf{S}, \mu) .
$$

Observe that, with $\mathbf{S}_{\mu}$ denoting the class of sets of $\mathbf{S}$ having finite $\mu$-measure as in (4.1), $\mathbf{S}\left(\mathbf{S}_{\mu}\right)$ is precisely the class of $\sigma$-finite subsets of $\mathbf{S}$. Since $\mu$ extends its restriction to $\mathbf{S}_{\mu}$, we have $\mu(A)=I_{\mu}\left(\chi_{A}\right)$ for any $A \in \mathbf{S}\left(\mathbf{S}_{\mu}\right)$ (Theorem (4.4)). Hence, by (5.1) and (3.3), we have

$$
\int s d \mu=I_{\mu} s \text { for any } s \in L\left[\mathbf{S}\left(\mathbf{S}_{\mu}\right)\right]^{+} .
$$


The verification that $\left(X, \mathscr{L}(\mathbf{S}, \mu), \int \cdot d \mu\right)$ is an integral space can now be carried out by elementary means (see [6] Chapter 11, Section 3 ) or by the following informative argument.

(5.5) Theorem. $\left(X, \mathscr{L}(\mathbf{S}, \mu), \int \cdot d \mu\right)$ coincides with $\left(X, \mathscr{L}_{\mu}[\mathrm{S}], I_{\mu}\right)$ of $\S 4$; it is thus the (unique) minimal integral space which extends the elementary integral space $\left(X, L\left[\mathbf{S}_{\mu}\right], \int \cdot d \mu\right)$.

Proof. It suffices to consider nonnegative functions. Suppose first that $f \in \mathscr{L}(\mathbf{S}, \mu)^{+}$. Then $f \in \mathscr{M}_{\mathrm{S}}^{+}$by definition, so there exist $s_{n} \in L[\mathrm{~S}]^{+}$such that $s_{n} \nearrow f$ (Proposition (2.5)). In view of definition (5.2), we have $\int s_{n} d \mu \leqq \int f d \mu<\infty$, and this (by (5.1)) implies that we actually have $s_{n} \in L\left[\mathrm{~S}_{\mu}\right]^{+}$. Thus, applying (5.4) and the Monotone Convergence Theorem (3.1) to the integral $I_{\mu}$ yields

$\left(^{*}\right) f \in \mathscr{L}(\mathbf{S}, \mu)^{+} \Rightarrow f \in \mathscr{L}_{\mu}[\mathbf{S}]^{+}$and $I_{\mu} f=\lim _{n} I_{\mu} s_{n}=\lim _{n} I_{\mu} s_{n}=\lim _{n} \int s_{n} d \mu \leqq \int f d \mu$.

On the other hand, if $f \in \mathscr{L}_{\mu}[\mathrm{S}]^{+}$, then $f \in \mathscr{M}_{\mathrm{S}\left(\mathrm{S}_{\mu}\right)}^{+}$by (4.3); so $f$ is certainly S-measurable. Suppose that $f \geqq s \in L[\mathrm{~S}]^{+}$, as in (5.2). Since $s \leqq f \in \mathscr{M}_{\mathrm{S}\left(\mathrm{S}_{\mu}\right)}^{+}$, we see that $s^{-1}(c, \infty]=s^{-1}(c$, $\infty] \cap f^{-1}(c, \infty]$, which is a $\sigma$-finite subset of $\mathbf{S}$; so we actually have $s \in L\left[\mathbf{S}\left(\mathbf{S}_{\mu}\right)\right]^{+}$. Applying (5.4) and the monotonicity of the integral $I_{\mu}$ yields

$$
\left({ }^{* *}\right) f \in \mathscr{L}_{\mu}[\mathbf{S}]^{+} \Rightarrow f \in \mathscr{L}(\mathbf{S}, \mu)^{+} \text {and } \int f d \mu=\sup \left\{I_{\mu} s: f \geqq s \in L\left[\mathbf{S}\left(\mathbf{S}_{\mu}\right)\right]\right\} \leqq I_{\mu} f
$$

The desired conclusion obtains upon combining $\left({ }^{*}\right)$ with $(* *)$.

6. Integrals that can be associated with measures. We now reverse the roles of measure and integral, and assume that we are given an integral space $(X, \mathscr{L}, I)$. For $(X, \mathscr{L}, I)$ to be associated with a measure, it is clearly necessary that

$$
f \in \mathscr{L} \Rightarrow f \wedge 1 \in \mathscr{L} .
$$

Stone ([8] II, pp. 452-454) showed how the integral resulting from a Daniell extension (3.4(a)) of an elementary integral space $(X, L, I)$ can be obtained from a (necessarily complete) measure provided $f \in L \Rightarrow f \wedge 1 \in L$. We now show how an arbitrary (not necessarily constructed) integral $I$ on $\mathscr{L}$ determines a minimal (not necessarily complete) measure space from which it can be retrieved by means of $\S 5$ whenever (6.1) holds.

(6.2) Theorem. If $(X, \mathscr{L}, I)$ is an integral space and $\mathscr{L}$ satisfies $(6.1)$, then the class $\mathbf{S}\{\mathscr{L}\}=\left\{A \subset X: \chi_{A} \in \mathscr{B}_{\mathscr{L}}\right\}$ is the smallest $\sigma$-ring with respect to which every function in $\mathscr{L}$ is measurable, and the set function $\mu_{I}$ defined on $\mathbf{S}\{\mathscr{L}\}$ by

$$
\mu_{I}(A)=I\left(\chi_{A}\right) \text { for } A \in \mathbf{S}\{\mathscr{L}\}
$$

(where $I\left(\chi_{A}\right)=\infty$ if $\left.\chi_{A} \in \mathscr{B}_{\mathscr{L}}^{+} \mid \mathscr{L}^{+}\right)$is a $\sigma$-finite measure on $\mathbf{S}\{\mathscr{L}\}$. Call $\left(X, \mathbf{S}\{\mathscr{L}\}, \mu_{I}\right)$ the measure space associated with $(X, \mathscr{L}, I)$. Then the minimal integral space associated with $\left(X, \mathbf{S}\{\mathscr{L}\}, \mu_{I}\right)$, viz. $\left(X, \mathscr{L}\left(\mathbf{S}\{\mathscr{L}\}, \mu_{I}\right), \int \cdot d \mu_{I}\right)$, coincides with the given integral space $(X, \mathscr{L}, I)$. 
Proof. It is clear that $\mathscr{L} \cap R^{X}$ is a Riesz space. Since (6.1) holds, a "truncation" argument shows that $\mathscr{B}_{\mathscr{L}}$ is precisely the class of $\mathscr{L} \cap R^{X}$-Baire functions; so $S\{\mathscr{L}\}$ is a $\sigma$-ring, by Lemma (2.2). That any $f \in \mathscr{L}^{+}$is $\mathbf{S}\{\mathscr{L}\}$-measurable follows from (6.1) and the fact that

$$
\mathscr{L} \ni[n(f-f \wedge c)] \wedge 1 \nearrow \chi_{f^{-1}(c, \infty)} \in \mathscr{B}_{L}, \text { i.e., } f^{-1}(c, \infty] \in \mathbf{S}\{L\}
$$

for any $c \in(0, \infty)$. But for any $\sigma$-ring $\mathrm{S}$ such that $\mathscr{L} \subset \mathscr{M}_{\mathrm{S}}$ we have $\mathscr{B}_{\mathscr{L}} \subset \mathscr{M}_{\mathrm{S}}$ by (2.6), so that $\mathbf{S}\{\mathscr{L}\} \subset \mathbf{S}$, i.e.

$\mathbf{S}\{\mathscr{L}\}$ is the smallest $\sigma$-ring $\mathbf{S}$ such that $\mathscr{L} \subset \mathscr{M}_{\mathrm{S}}$

Clearly $\mu_{I}$ is a measure on $\mathbf{S}\{\mathscr{L}\}$. Let $\mathbf{R}\{\mathscr{L}\}=\left\{A \in \mathbf{S}\{\mathscr{L}\}: \chi_{A} \in \mathscr{L}\right\}$. Then (3.2) implies that for any $A \in \mathbf{S}\{\mathscr{L}\}$, either $A \in \mathbf{R}\{\mathscr{L}\}$ or there exist $f_{n} \in \mathscr{L}^{+}$such that $f_{n} \nearrow \chi_{A}$. In the latter case, taking $A_{n}=f^{-1}\left(\frac{1}{2}, \infty\right]$ gives $A=\bigcup_{n} A_{n}$ and $A_{n} \in \mathbf{R}\{\mathscr{L}\}$ (by (3.2) again, since $\left.\chi_{A_{n}} \leqq 2 f \in \mathscr{L}\right)$. Hence $\mu_{I}$ is $\sigma$-finite and $\mathbf{S}\{\mathscr{L}\}=\mathbf{S}(\mathbf{R}\{\mathscr{L}\})$. But both the given integral $I$ and the integral $\int \cdot d \mu_{I}$ (associated with $\left(X, \mathbf{S}\{\mathscr{L}\}, \mu_{I}\right)$ in $\left.\S 5\right)$ agree on the Riesz space $L[\mathbf{R}\{\mathscr{L}\}]$. Since $\mathscr{L}^{+} \cap \mathscr{L}\left(\mathbf{S}\{\mathscr{L}\}, \mu_{I}\right) \subset \mathscr{M}_{\mathrm{S}}^{+}=\mathscr{B}_{L[R\{\mathscr{L}\}]}^{+}$by (2.5), we conclude from Theorem (3.2) that $(X, \mathscr{L}, I)$ and $\left(X, \mathscr{L}\left(\mathbf{S}\{\mathscr{L}\}, \mu_{I}\right), \int \cdot d \mu_{I}\right)$ coincide.

Combining this with $\S 5$, we obtain the main result we seek:

(6.3) Theorem. The map $\phi:(X, \mathscr{L}, I) \rightarrow\left(X, \mathbf{S}\{\mathscr{L}\}, \mu_{I}\right)$ described in (6.2) establishes a 1-1 correspondence between the collection of all integral spaces $(X, \mathscr{L}, I)$ for which $(6.1)$ holds and the collection of all $\sigma$-finite measure spaces $(X, \mathbf{S}, \mu)$. Its inverse is the map $(X, \mathbf{S}, \mu) \rightarrow$ $\left(X, \mathscr{L}(\mathbf{S}, \mu), \int \cdot d \mu\right)$ described in $\S 5$.

(6.4) COROLlaRY. By restricting $\phi$ of (6.3), we obtain a 1-1 correspondence between the collection of all I-complete (3D of [5]) integral spaces $(X, \mathscr{L}, I)$ satisfying (6.1) and the collection of all complete $\sigma$-finite measure spaces $(X, \mathrm{~S}, \mu)$.

7. Discussion. Segal and Kunze $([7], \S 3.2)$ define the functions measurable with respect to $\mathbf{R}$ to be $\mathscr{B}_{L[\mathbf{R}]}$ (cf. $(2.5)$ ). Bogdanowicz [1] characterized $\left(X, \mathscr{L}(\mathbf{S}, \mu) \cap R^{X}, \int \cdot d \mu\right)$ as a minimal integral extension as in (5.5); but his argument assumes that this triple is an integral space, whereas our argument proves it.

In their lecture notes, which were kindly sent to me by the authors, Kelly and Srinnivasan [3] obtain a result similar to Theorem (6.2), namely (in the notation of (6.2)) that to each integral $I$ on the function lattice $\mathscr{L}$ (identified with $\mathscr{L} \cap R^{X}$ ) which satisfies (6.1) there is a measure on a $\delta$-ring (namely $\mu_{I}$ on $\mathbf{R}\{\mathscr{L}\}$, in the notation of $(6.2)$ ) from which $\left(X, \mathscr{L} \cap R^{X}, I\right)$ can be retrieved. However, they observe that not all measures $\mu$ on a $\delta$-ring (i.e. a ring closed under countable intersection) can be obtained this way; so their surjective map $(X, \mathbf{R}, \mu) \rightarrow$ $\left(X, \mathscr{L} \cap R^{X}, I\right)$ is not injective. Using the results of Stone [8], Zaanen ([9], Theorems 7 and 9 of $\S 17$ and Problem 32.13) in effect obtains Corollary (6.4); but his argument cannot be modified to give the much sharper result (6.3). 


\section{REFERENCES}

1. W. M. Bogdanowicz, An approach to the theory of integration generated by positive line functionals and existence of minimal extensions. Proc. Japan Acad. 43 (1967) 186-191.

2. P. R. Halmos, Measure Theory (Van Nostrand Company, New Jersey, 1950).

3. J. L. Kelly and T. P. Srinnivasan (Lecture Notes, University of Berkeley).

4. L. H. Loomis, An Introduction to Abstract Harmonic Analysis (Van Nostrand Company, New Jersey, 1953).

5. M. J. Maron, Abstract integral spaces and minimal extensions, Glasgow Math. J. 12 (1971), $166-178$

6. H. L. Royden, Real Analysis, Second Edition (Macmillan Company, New York, 1968).

7. I. E. Segal and R. A. Kunze, Integrals and Operators (McGraw-Hill Book Company, New York, 1968).

8. M. H. Stone, Notes on Integration. Proc. Nat. Acad. Sci. U.S.A. 34 (1948), (I) 336-342, (II) 447-455, (III) 483-490, 35 (1949), (IV) 50-58. 1967).

9. A. C. Zaanen, Integration, Second Edition (North-Holland Publishing Company, Amsterdam,

\section{UNIVERSITY OF LOUISVILLE}

